\title{
Monitor-based exoscopic 3D4k neurosurgical interventions: a two-phase prospective-randomized clinical evaluation of a novel hybrid device
}

\author{
Anna L. Roethe ${ }^{1,2}$ (D) $\cdot$ Philipp Landgraf ${ }^{3} \cdot$ Torsten Schröder $^{3} \cdot$ Martin Misch $^{1} \cdot$ Peter Vajkoczy $^{1} \cdot$ Thomas Picht $^{1,2}$
}

Received: 7 November 2019 / Accepted: 18 April 2020 / Published online: 19 May 2020

(C) The Author(s) 2020

\begin{abstract}
Background Promoting a disruptive innovation in microsurgery, exoscopes promise alleviation of physical strain and improved image quality through digital visualization during microneurosurgical interventions. This study investigates the impact of a novel 3D4k hybrid exoscope (i.e., combining digital and optical visualization) on surgical performance and team workflow in preclinical and clinical neurosurgical settings.

Methods A pre-clinical workshop setting has been developed to assess usability and implementability through skill-based scenarios (neurosurgical participants $n=12$ ). An intraoperative exploration in head and spine surgery $(n=9)$ and a randomized clinical study comparing ocular and monitor mode in supratentorial brain tumor cases $(n=20)$ followed within 12 months. Setup, procedure, case characteristics, surgical performance, and user experience have been analyzed for both ocular group (OG) and monitor group (MG).

Results Brain tumor cases using frontal, frontoparietal, or temporal approaches have been identified as favorable use cases for introducing exoscopic neurosurgery. Mean monitor distance and angle were $180 \mathrm{~cm}$ and $10^{\circ}$. Surgical ergonomics when sitting improved significantly in MG compared with OG $(P=.03)$. Hand-eye coordination required familiarization in MG. Preclinical data showed a positive correlation between lateral camera inclination and impact on hand-eye coordination $\left(r_{\mathrm{s}}=0.756, P=.01\right)$. There was no significant added surgical time in MG. Image quality in current generation 3D4k monitors has been rated inferior to optic visualization yet awaits updates.

Conclusions The hybrid exoscopic device can be integrated into established neurosurgical workflows. Currently, exoscopic interventions seem most suited for cranial tumor surgery in lesions that are not deep-seated. Ergonomics improve in monitor mode compared to conventional microsurgery.
\end{abstract}

Keywords Brain tumor $\cdot$ Digital innovation $\cdot$ Exoscope $\cdot$ Intraoperative visualization $\cdot$ Technology evaluation

This article is part of the Topical Collection on CSF Neurosurgical technique evaluation

Selected results of this research have been previously presented at the annual meeting of the German neurosurgical society in Münster, Germany, June 4, 2018 (doi: 10.3205/18dgnc387) and in Würzburg, Germany, May 13, 2019 (doi: 10.3205/19dgnc091).

German Clinical Trials Register No DRKS00016674

Electronic supplementary material The online version of this article (https://doi.org/10.1007/s00701-020-04361-2) contains supplementary material, which is available to authorized users.

Anna L. Roethe

anna.roethe@charite.de

Extended author information available on the last page of the article

\section{Introduction}

Microsurgery is being facing a new trend: the convergence of endoscopic and microscopic visualization principles resulting in lighter and more versatile surgical exoscopes. From the clinical perspective, this device evolution is designed to address current constraints in surgical access and ergonomics, digitally enhanced visualization and information sharing in the OR, all of which can have high impact on surgical interventions of varying complexity. Exoscope technology is trying to overcome previously experienced limitations in endoscopy and surgical microscopy $[25,26]$ by merging these two major intraoperative visualization techniques $[4,22]$ and seeking to establish new quality standards [4]. An exoscope is an extracorporeal scope [13] distanced from the surgical site, 
producing high-quality video images with a wide field of view [19]. Its components comprise a tubular telescope or microscope, a camera unit, a light source, a monitor, and a control unit. For many exoscopes directly base on the principles of endoscopy, they reproduce its inherent dissociation of working and viewing direction. In return, these machines promise to facilitate improvements in OR team visualization and surgical ergonomics [29]. Preclinical and clinical data is available for six exoscopic devices by different manufacturers (see Table 1), all of which benefit from recent technology improvements. However, disruptive effects on surgical workflow and intraoperative visualization requirements remain topics yet to be addressed more thoroughly. The introduction of the first digital hybrid visualization device combining exoscopic and microscopic features allowed for direct comparison of conventional and exoscopic surgery. To assist its clinical investigation, we set up a mixed method-study design focusing on identification of eligible cases, assessment of usability, analysis of device impact on surgical performance and team workflows, and exploration of visual quality standards in microneurosurgery.

\section{Methods and materials}

\section{System description}

The hybrid visualization system assessed in this study (KINEVO 900, Carl Zeiss Meditec AG, Oberkochen, Germany) can be utilized as a conventional (optical) surgical microscope or as an exoscope with digital visualization on an external 55" 3D4k monitor plus an internal 24" 3DHD system monitor (utilizing passive 3D glasses). System specifications include a working distance of 200-625 mm, focal length of $170 \mathrm{~mm}$, maximum magnification in exoscope mode $11, \times 6$, $\mathrm{XY}$ robotic movement in 6 axes and 3D4k stereo video cameras ( $2 \times 3$-chip $4 \mathrm{~K}, 2160$ p). Robotics can be controlled via a wireless 10-button plus joystick foot control panel (FCP).

\section{Study design and randomization}

The investigation addressed both quantitative and qualitative aspects of the device utilization in two phases (preclinical/ clinical). In a two-day preclinical workshop in an actual OR environment, 12 neurosurgical professionals ( 6 residents PGY-2 to 5 and 6 attendings) performed predefined tasks on a skull/brain and spine phantom in a simulated team setting (see Fig. 1a-b). The tasks involved microsurgical vessel preparation and anastomosis (I) or tissue and nerve preparation (II) in a chicken wing/thigh model fixated in a skull simulator (Neuro Patient `Graf^, Phacon, Leipzig, Germany), as well as lumbar decompression (III) in a spine simulator (Lumbar Spine Patient `Schumann`, Phacon, Leipzig, Germany). Setup and task performance have been documented in each participant followed by a questionnaire with a 4-point Likert scale (1: excellent, 4: not acceptable) containing 30 items total (see supplementary material). The questionnaire results served as a basis for the definition of items in study phase 2 . All items have been weighted equally.

After clinical introduction, the exoscope has been employed in a range of neurosurgical interventions (see Table 2, Fig. 1c). User experience has been rated on an adjusted 5-point Likert scale (1: excellent, 5: not acceptable) regarding setup and ergonomics, workflow, usability, and image quality. The randomized study phase consisted of a parallel group trial with 1:1 allocation ratio (equal numbers of prepared tickets for both groups); the same expert neurosurgeons $(n=4)$ performed either conventional or exoscopic procedures. A series of 20 subsequent uncomplicated supratentorial brain tumors has been randomly assigned to either monitor group (MG) or ocular group (OG) within $24 \mathrm{~h}$ before intervention. The study team enrolled and assigned patients; further inclusion criteria were no challenging microneurosurgical tasks involved, little expected blood loss, no to mild multimorbidity (ASA 1-2), age $>18$ years. Switching to the other operating mode respectively has been optional at any time. The intraoperative documentation included microscope recording, setup photos, a structured observer assessment sheet containing pre-defined items plus a short interview allowing for additional user comments. Assessment parameters were surgical time, conversion of modality, ergonomics, surgical performance, setup specifications, workflow, and satisfaction with image quality. We used validated questionnaires from the System Usability Scale (SUS) [8] to assess different dimensions of usability and the Surgery Task Load Index (SURG-TLX) [30] to measure surgical workload. Ethical approval has been obtained from the local IRB (EA2/081/18), and written consent from both patients and surgeon participants was collected prior to intervention. The study has been registered with the German Clinical Trials Register (DRKS00016674). Results of the randomized clinical evaluation are reported according to the 2017 CONSORT NPT extension [7].

\section{Metrics of surgical performance}

3D stereoscopic vision [5] has been tested in all participants with a commercially available and validated stereopsis screening test (Frisby Pocket Stereo Test, Stereotest Ltd., Fulwood, UK, 2013). For assessment of surgical performance, we combined quantitative performance data (surgical time, EOR, complications, functional outcome) with semi-quantitative and qualitative performance data (surgical task completion, usability experience), using video analysis, scientific observation, and self-assessment. For investigation of potential impact on hand-eye coordination, particularly in the endoscopically 
Table 1 Preclinical and clinical investigation of exoscopic devices in neurosurgery 2008-2018 $(n=23)$

\begin{tabular}{|c|c|c|c|c|c|c|}
\hline Year & Paper & $\begin{array}{l}\text { Type of } \\
\text { study }\end{array}$ & Exoscope & Device characteristics & Type of surgery $(n)$ & Evaluation of exoscope \\
\hline 2018 & $\begin{array}{l}\text { Takahashi et al. } \\
\text { [29] }\end{array}$ & Clinical & $\begin{array}{l}\text { ORBEYE (Olympus, Tokyo, } \\
\text { Japan) }\end{array}$ & $\begin{array}{l}\text { 3D4k, 55" monitor, 3D } \\
\text { glasses, working } \\
\text { distance } 22-55 \mathrm{~cm} \text {, } \\
\text { FCP or manual control }\end{array}$ & $\begin{array}{l}\text { Cranial (tumor, } \\
\text { neurovascular) } \\
(n=14)\end{array}$ & $\begin{array}{l}\text { (+) Setup, size, } \\
\text { ergonomics, } \\
\text { combination with } \\
\text { navigation, HQ visuals } \\
\text { for entire team } \\
\text { (-) Integration of surgical } \\
\text { assistant (rotated view) }\end{array}$ \\
\hline 2018 & Kwan et al. [17] & Clinical & $\begin{array}{l}\text { ORBEYE (Olympus, Tokyo, } \\
\text { Japan) }\end{array}$ & $\begin{array}{l}\text { 3D4k, 55" monitor, 3D } \\
\text { glasses, working } \\
\text { distance } 22-55 \mathrm{~cm} \text {, } \\
\text { FCP or manual control }\end{array}$ & $\begin{array}{l}\text { Spinal (mainly } \\
\text { laminectomies) } \\
(n=10)\end{array}$ & $\begin{array}{l}\text { (+) Ergonomics, size, } \\
\text { weight, } \\
\text { maneuverability, single } \\
\text { hand manipulation, } \\
\text { depth of field, } \\
\text { immersion, HQ visuals } \\
\text { for entire team, } \\
\text { education } \\
\text { (-) Scope adjustments, } \\
\text { controls, case length, } \\
\text { learning curve, monitor } \\
\text { positioning when } \\
\text { additional equipment }\end{array}$ \\
\hline 2018 & Belykh et al. [4] & $\begin{array}{l}\text { Preclinical } \\
\quad \text { (labora- } \\
\text { tory } \\
\text { study) }\end{array}$ & $\begin{array}{l}\text { ROVOT-M/ BRIGHTMATTER } \\
\text { SERVO (Synaptive Medical, } \\
\text { Toronto, Ontario, Canada) / } \\
\text { KINEVO } 900 \text { (Carl Zeiss AG, } \\
\text { Oberkochen, Germany) }\end{array}$ & $\begin{array}{l}\text { 2DHD, 55" monitor, } \\
\text { working distance } \mathrm{n} / \mathrm{A} \text {, } \\
\text { manual control + FCP / } \\
\text { 3D4k, 55" monitor, } \\
\text { working distance } \\
\text { 20-62,5 cm, FCP or } \\
\text { manual control }\end{array}$ & $\begin{array}{l}\text { Neurovascular } \\
\text { techniques }\end{array}$ & $\begin{array}{l}\text { KINEVO } 900 \\
\text { (+) 3D Depth perception } \\
\text { (-) Depth perception and } \\
\text { details at high } \\
\text { magnification, } \\
\text { physiologic eye } \\
\text { accommodation, field } \\
\text { of view } \\
\text { ROVOT-M } \\
\text { (+) Size } \\
\text { (-) Stereopsis } \\
\text { both } \\
\text { (+) High resolution, } \\
\text { ergonomics, education } \\
\text { (-) 3D glasses, slight } \\
\text { delay, learning curve }\end{array}$ \\
\hline 2018 & $\begin{array}{l}\text { Bakhsheshian } \\
\text { et al. [1] }\end{array}$ & Clinical & $\begin{array}{l}\text { VITOM (KARL } \\
\text { STORZ-Endoscopy America, } \\
\text { Inc., El Segundo, California, } \\
\text { USA) / ROVOT-M/ } \\
\text { BRIGHTMATTER SERVO } \\
\text { (Synaptive Medical, Toronto, } \\
\text { Ontario, Canada) }\end{array}$ & $\begin{array}{l}\text { 2DHD, 0- or 90-degree, } \\
26^{\prime \prime} \text { monitor, working } \\
\text { distance } 25-75 \mathrm{~cm}, \\
\text { manual control/2DHD, } \\
55^{\prime \prime} \text { monitor, working } \\
\text { distance n/A, manual } \\
\text { control + FCP }\end{array}$ & $\begin{array}{l}\text { Cranial (subcortical } \\
\text { brain metastases) } \\
\quad(n=25)\end{array}$ & $\begin{array}{l}\text { (+) Depth of field, } \\
\text { ergonomics } \\
(-) \text { None }\end{array}$ \\
\hline 2018 & Gassie et al. [9] & Clinical & $\begin{array}{l}\text { VITOM (Karl Storz Endoscopy } \\
\text { America, Inc., El Segundo, } \\
\text { California, USA) }\end{array}$ & $\begin{array}{l}\text { 2DHD, } 0 \text { - or } 90 \text {-degree, } \\
26^{\prime \prime} \text { monitor, working } \\
\text { distance } 25-75 \mathrm{~cm}, \\
\text { manual control }\end{array}$ & $\begin{array}{l}\text { Cranial (subcortical } \\
\text { tumors) }(n=50)\end{array}$ & $\begin{array}{l}\text { (+) Ergonomics, wider } \\
\text { field of view } \\
(-) \text { Visualization angle, } \\
\text { depth interpretation, } \\
\text { micro adjustments of } \\
\text { working field }\end{array}$ \\
\hline 2018 & Beez et al. [2] & Clinical & $\begin{array}{l}\text { VITOM 3D (Karl Storz GmbH, } \\
\text { Tuttlingen, Germany) }\end{array}$ & $\begin{array}{l}\text { 3D4k, 32" monitor, 3D } \\
\text { glasses, working } \\
\text { distance } 20-50 \mathrm{~cm} \text {, } \\
\text { manual control } \\
\text { (Joystick) }\end{array}$ & $\begin{array}{l}\text { Cranial and spinal, } \\
\text { pediatric } \\
\text { neurosurgery } \\
\text { (lesion, tumor, } \\
\text { myelomeningoc- } \\
\text { ele) }(n=3)\end{array}$ & $\begin{array}{l}\text { (+) Ergonomics, } \\
\text { accessibility of the } \\
\text { surgical field, } \\
\text { immersion, size, cost } \\
\text { savings, HQ visuals for } \\
\text { entire team, education } \\
\text { (-) Deep lesions, } \\
\text { illumination, lateral } \\
\text { inclination of scope, } \\
\text { adjustment controls, }\end{array}$ \\
\hline
\end{tabular}


Table 1 (continued)

\begin{tabular}{|c|c|c|}
\hline Year Paper & Type of & Exoscope \\
\hline
\end{tabular}
study

\begin{tabular}{|c|c|c|c|c|c|c|}
\hline & & & & & & $\begin{array}{l}\text { user-friendliness, } \\
\text { assistant integration }\end{array}$ \\
\hline 2018 & Klinger et al. [15] & Clinical & $\begin{array}{l}\text { ROVOT-M/ BRIGHTMATTER } \\
\text { SERVO (Synaptive Medical, } \\
\text { Toronto, Ontario, Canada) }\end{array}$ & $\begin{array}{l}\text { 2DHD, 55" monitor, } \\
\text { working distance } \mathrm{n} / \mathrm{A}, \\
\text { manual control + FCP }\end{array}$ & $\begin{array}{l}\text { Cranial (aneurysm) } \\
\quad(n=1)\end{array}$ & $\begin{array}{l}\text { (+) Ergonomics, size, } \\
\text { maneuverability, } \\
\text { unobstructed surgical } \\
\text { field, visualization of } \\
\text { difficult angles/ } \\
\text { trajectories, HQ visuals } \\
\text { for entire team, ease of } \\
\text { use, magnification, } \\
\text { education } \\
\text { (-) Steep learning curve, } \\
\text { stereopsis, depth } \\
\text { perception }\end{array}$ \\
\hline 2018 & Sack et al. [26] & $\begin{array}{l}\text { Preclinical } \\
\text { (cadaver } \\
\text { study) }\end{array}$ & $\begin{array}{l}\text { ORBEYE (Olympus, Tokyo, } \\
\text { Japan) }\end{array}$ & $\begin{array}{l}\text { 3D4k, } 55^{\prime \prime} \text { monitor, 3D } \\
\text { glasses, working } \\
\text { distance } 22-55 \mathrm{~cm} \text {, } \\
\text { FCP or manual control }\end{array}$ & $\begin{array}{l}\text { Cranial (exposure } \\
\text { and dissection) } \\
(n=7)\end{array}$ & $\begin{array}{l}\text { (+) Optics, depth of field, } \\
\text { ergonomics, size, single } \\
\text { hand manipulation, no } \\
\text { balancing required, } \\
\text { maneuverability, } \\
\text { immersion, HQ visuals } \\
\text { for entire team, } \\
\text { education, cost savings } \\
\text { (-) Learning curve, } \\
\text { monitor setup, blocking } \\
\text { of line of sight by } \\
\text { device }\end{array}$ \\
\hline 2017 & Rossini et al. [25] & Clinical & $\begin{array}{l}\text { VITOM 3D (Karl Storz GmbH, } \\
\text { Tuttlingen, Germany) }\end{array}$ & $\begin{array}{l}\text { 3D4k, 32" monitor, 3D } \\
\text { glasses, working } \\
\text { distance } 20-50 \mathrm{~cm} \text {, } \\
\text { manual control } \\
\text { (Joystick) }\end{array}$ & $\begin{array}{l}\text { Cranial } \\
\qquad(\text { meningioma }) \\
(n=1)\end{array}$ & $\begin{array}{l}\text { (+) Ergonomics, size, } \\
\text { maneuverability, } \\
\text { weight, depth of field, } \\
\text { 3D vision, color filter, } \\
\text { magnification, } \\
\text { microscopic-- } \\
\text { macroscopic switch, } \\
\text { HQ visuals for entire } \\
\text { team, education, cost } \\
\text { savings } \\
\text { (-) Repositioning, } \\
\text { refocusing, } \\
\text { magnification, second } \\
\text { screen needed, controls }\end{array}$ \\
\hline 2017 & $\begin{array}{l}\text { Oertel/Burkhardt } \\
\text { [23] }\end{array}$ & Clinical & $\begin{array}{l}\text { VITOM 3D (Karl Storz GmbH, } \\
\text { Tuttlingen, Germany) }\end{array}$ & $\begin{array}{l}\text { 3D4k, 32" monitor, 3D } \\
\text { glasses, working } \\
\text { distance } 20-50 \mathrm{~cm} \text {, } \\
\text { manual control } \\
\text { (Joystick) }\end{array}$ & $\begin{array}{l}\text { Cranial and spinal } \\
\text { (tumor, fusion, } \\
\text { decompression, } \\
\text { discectomy) } \\
(n=16)\end{array}$ & $\begin{array}{l}\text { (+) Ergonomics, size, } \\
\text { maneuverability, depth } \\
\text { of field, cost savings, } \\
\text { education } \\
\text { (-) Repositioning, } \\
\text { identification of } \\
\text { bleeding vessels }\end{array}$ \\
\hline 2017 & Nishiyama [22] & Review & $\begin{array}{l}\text { VITOM (Karl Storz Endoscopy } \\
\text { America, Inc., El Segundo, } \\
\text { California, USA) }\end{array}$ & $\begin{array}{l}\text { 2DHD, 0- or 90-degree, } \\
26^{\prime \prime} \text { monitor, working } \\
\text { distance } 25-75 \mathrm{~cm}, \\
\text { manual control }\end{array}$ & $\begin{array}{l}\text { Spinal (lipoma) } \\
\quad(n=1)\end{array}$ & $\begin{array}{l}\text { (+) Wide field of view, } \\
\text { deep focus, less } \\
\text { repositioning } \\
\text { (-) Magnification, digital } \\
\text { zoom, stereopsis }\end{array}$ \\
\hline 2017 & Moisi et al. [21] & $\begin{array}{l}\text { Preclinical } \\
\quad \text { (cadaver } \\
\text { study) }\end{array}$ & $\begin{array}{l}\text { ROVOT-M/ BRIGHTMATTER } \\
\text { SERVO (Synaptive Medical, } \\
\text { Toronto, Ontario, Canada) }\end{array}$ & $\begin{array}{l}\text { 2DHD, 55" monitor, } \\
\text { working distance } \mathrm{n} / \mathrm{A}, \\
\text { manual control + FCP }\end{array}$ & $\begin{array}{l}\text { Spinal (unilateral, } \\
\quad \text { single-level } \\
\text { laminotomies) } \\
(n=22)\end{array}$ & $\begin{array}{l}\text { (+)Ergonomics, depth of } \\
\text { field, maneuverability, } \\
\text { education, no added } \\
\text { time } \\
\text { (-) Stereopsis }\end{array}$ \\
\hline 2017 & $\begin{array}{l}\text { Jackson et al. } \\
\text { [13] }\end{array}$ & Clinical & & $\begin{array}{l}\text { 2DHD, 0- or } 90 \text {-degree, } \\
26^{\prime \prime} \text { monitor, working }\end{array}$ & & $\begin{array}{l}\text { (+) Bimanual } \\
\text { manipulation, }\end{array}$ \\
\hline
\end{tabular}


Table 1 (continued)

\begin{tabular}{|c|c|c|c|c|c|c|}
\hline Year & Paper & $\begin{array}{l}\text { Type of } \\
\text { study }\end{array}$ & Exoscope & Device characteristics & Type of surgery $(n)$ & Evaluation of exoscope \\
\hline & & & $\begin{array}{l}\text { VITOM (Karl Storz Endoscopy } \\
\text { America, Inc., El Segundo, } \\
\text { California, USA) }\end{array}$ & $\begin{array}{l}\text { distance } 25-75 \mathrm{~cm} \text {, } \\
\text { manual control }\end{array}$ & $\begin{array}{l}\text { Cranial (deep seated } \\
\text { lesions with } \\
\text { biopsy) }(n=11)\end{array}$ & $\begin{array}{l}\text { flexibility, higher } \\
\text { magnification } \\
(-) \text { None }\end{array}$ \\
\hline 2017 & $\begin{array}{l}\text { Krishnan et al. } \\
\text { [16] }\end{array}$ & Clinical & $\begin{array}{l}\text { VITOM (Karl Storz Endoscopy } \\
\text { America, Inc., El Segundo, } \\
\text { California, USA) }\end{array}$ & $\begin{array}{l}\text { 2DHD, 0- or 90-degree, } \\
26^{\prime \prime} \text { monitor, working } \\
\text { distance } 25-75 \mathrm{~cm}, \\
\text { manual control }\end{array}$ & $\begin{array}{l}\text { Cranial and spinal } \\
\text { (decompression, } \\
\text { laminotomy, } \\
\text { tumor, ICH) } \\
(n=18)\end{array}$ & $\begin{array}{l}\text { (+) Ergonomics, size, } \\
\text { magnification, } \\
\text { illumination, HD } \\
\text { images, angulation, } \\
\text { depth of field } \\
\text { (-) Adjustment and } \\
\text { refocusing, added time, } \\
\text { fluorescence filters, } \\
\text { navigation, (stereopsis) }\end{array}$ \\
\hline 2017 & Gonen et al. [11] & Clinical & $\begin{array}{l}\text { ROVOT-M/BRIGHTMATTER } \\
\text { SERVO (Synaptive Medical, } \\
\text { Toronto, Ontario, Canada) }\end{array}$ & $\begin{array}{l}\text { 2DHD, 55" monitor, } \\
\text { working distance n/A, } \\
\text { manual control + FCP }\end{array}$ & $\begin{array}{l}\text { Cranial (tumors and } \\
\text { ICH) }(n=200)\end{array}$ & $\begin{array}{l}\text { (+) No complications, } \\
\text { robotic control } \\
(-) \text { Learning curve, } \\
\text { stereopsis }\end{array}$ \\
\hline 2016 & $\begin{array}{l}\text { Kassam et al. } \\
\text { [14] }\end{array}$ & $\begin{array}{l}\text { Preclinical } \\
\text { (cadaver } \\
\text { study), } \\
\text { clinical }\end{array}$ & $\begin{array}{l}\text { ROVOT-M/BRIGHTMATTER } \\
\text { SERVO (Synaptive Medical, } \\
\text { Toronto, Ontario, Canada) }\end{array}$ & $\begin{array}{l}\text { 2DHD, 55" monitor, } \\
\text { working distance n/A, } \\
\text { manual control + FCP }\end{array}$ & $\begin{array}{l}\text { Cranial (aneurysms) } \\
\quad(n=2+6)\end{array}$ & $\begin{array}{l}\text { (+) Larger immersive } \\
\text { volume of surgical } \\
\text { anatomy, preset } \\
\text { positions, hand-free } \\
\text { control } \\
\text { (-) None }\end{array}$ \\
\hline 2014 & Piquer et al. [24] & Clinical & $\begin{array}{l}\text { HDXO-SCOPE (Karl Storz } \\
\text { Endoscopy, Tuttlingen, } \\
\text { Germany) }\end{array}$ & $\begin{array}{l}\text { 2DHD, 23" monitor, } \\
\text { working distance } \\
20 \mathrm{~cm} \text {, manual control }\end{array}$ & $\begin{array}{l}\text { Cranial (tumors) } \\
\quad(n=38)\end{array}$ & $\begin{array}{l}\text { (+) Ergonomics, reduction } \\
\text { of fatigue, fluorescence, } \\
\text { wide field, HQ visuals } \\
\text { for entire team, weight, } \\
\text { cost savings } \\
\text { (-) None }\end{array}$ \\
\hline 2014 & Birch et al. [6] & Clinical & $\begin{array}{l}\text { VITOM (Karl Storz Endoscopy, } \\
\text { Tuttlingen, Germany) }\end{array}$ & $\begin{array}{l}\text { 2DHD, 90-degree, } 23 " \\
\text { monitor, working } \\
\text { distance } 25-75 \mathrm{~cm} \text {, } \\
\text { manual control }\end{array}$ & $\begin{array}{l}\text { Cranial (pineal } \\
\quad \text { lesions) }(n=5)\end{array}$ & $\begin{array}{l}\text { (+) Depth of field, } \\
\text { ergonomics, HQ } \\
\text { visuals for entire team, } \\
\text { no added time } \\
\text { (-) Stereopsis, assistant } \\
\text { integration }\end{array}$ \\
\hline 2014 & Belloch et al. [3] & Clinical & $\begin{array}{l}\text { HDXO-SCOPE (Karl Storz } \\
\text { Endoscopy, Tuttlingen, } \\
\text { Germany) }\end{array}$ & $\begin{array}{l}\text { 2DHD, 23" monitor, } \\
\text { working distance } \\
20 \mathrm{~cm} \text {, manual control }\end{array}$ & $\begin{array}{c}\text { Cranial (HGG) } \\
\quad(n=23)\end{array}$ & $\begin{array}{l}\text { (+) Cost savings, agility, } \\
\text { weight, handling, } \\
\text { ergonomics, } \\
\text { microscopic-- } \\
\text { macroscopic switch, } \\
\text { HQ visuals for entire } \\
\text { team, sterilizable } \\
\text { (-) None }\end{array}$ \\
\hline 2012 & $\begin{array}{l}\text { Shirzadi et al. } \\
\text { [27] }\end{array}$ & Clinical & $\begin{array}{l}\text { VITOM (Karl Storz Endoscopy, } \\
\text { Tuttlingen, Germany) }\end{array}$ & $\begin{array}{l}\text { 2DHD, 0-degree, 23" } \\
\text { monitor, working } \\
\text { distance } 25-75 \mathrm{~cm} \text {, } \\
\text { manual control }\end{array}$ & $\begin{array}{l}\text { Spinal } \\
\text { (decompressions } \\
\text { and interbody } \\
\text { fusions) }(n=48)\end{array}$ & $\begin{array}{l}\text { (+) Optics, ergonomics, } \\
\text { size, weight, versatility, } \\
\text { interoperability, depth } \\
\text { of field, no added time, } \\
\text { HQ visuals for entire } \\
\text { team, education, } \\
\text { sterilizable } \\
\text { (-) Stereopsis, } \\
\text { repositioning }\end{array}$ \\
\hline 2012 & $\begin{array}{l}\text { Mamelak et al. } \\
\text { [20] }\end{array}$ & Clinical & $\begin{array}{l}\text { VITOM (Karl Storz Endoscopy, } \\
\text { Tuttlingen, Germany) }\end{array}$ & $\begin{array}{l}\text { 2DHD, 23" monitor, } \\
\text { working distance } \\
25-60 \mathrm{~cm} \text {, manual } \\
\text { control }\end{array}$ & $\begin{array}{l}\text { Cranial (pineal } \\
\text { lesion) }(n=1)\end{array}$ & $\begin{array}{l}\text { (+) Ergonomics, depth of } \\
\text { field } \\
(-) \text { Stereopsis, } \\
\text { repositioning, focusing }\end{array}$ \\
\hline 2010 & $\begin{array}{l}\text { Mamelak et al. } \\
\text { [19] }\end{array}$ & Clinical & $\begin{array}{l}\text { HDXO-SCOPE (Karl Storz } \\
\text { Endoscopy, Tuttlingen, } \\
\text { Germany) }\end{array}$ & $\begin{array}{l}\text { 2DHD, 23" monitor, } \\
\text { working distance } \\
20 \mathrm{~cm} \text {, manual control }\end{array}$ & $\begin{array}{l}\text { Cranial and spinal } \\
\quad(\text { mainly tumors }) \\
(n=16)\end{array}$ & $\begin{array}{l}\text { (+) Ergonomics, } \\
\text { magnification, HQ } \\
\text { visuals for entire team, }\end{array}$ \\
\hline
\end{tabular}


Table 1 (continued)

\begin{tabular}{|c|c|c|c|c|c|c|}
\hline Year & Paper & $\begin{array}{l}\text { Type of } \\
\text { study }\end{array}$ & Exoscope & Device characteristics & Type of surgery $(n)$ & Evaluation of exoscope \\
\hline & & & & & & $\begin{array}{l}\text { size, interoperability, } \\
\text { cost savings, education } \\
\text { (-) Stereopsis, } \\
\text { repositioning, focusing, } \\
\text { angulation, robotics }\end{array}$ \\
\hline 2008 & $\begin{array}{l}\text { Mamelak et al. } \\
{[18]}\end{array}$ & $\begin{array}{l}\text { Preclinical } \\
\text { (animal } \\
\text { model) }\end{array}$ & $\begin{array}{l}\text { HDXO-SCOPE (Karl Storz } \\
\text { Endoscopy, Tuttlingen, } \\
\text { Germany) }\end{array}$ & $\begin{array}{l}\text { 2DHD, 23" monitor, } \\
\text { working distance } \\
20 \mathrm{~cm} \text {, manual control }\end{array}$ & $\begin{array}{l}\text { Cranial (brain } \\
\text { dissection in live } \\
\text { pig model) } \\
(n=4)\end{array}$ & $\begin{array}{l}\text { (+) Ergonomics, } \\
\text { magnification, HQ } \\
\text { visuals for entire team, } \\
\text { size, interoperability, } \\
\text { cost, sterilizable } \\
\text { (-) Stereopsis, light } \\
\text { intensity, telescope arm } \\
\text { control, focus and } \\
\text { zoom }\end{array}$ \\
\hline
\end{tabular}

FCP foot control panel, $H G G$ high grade glioma, $H Q$ high quality, $I C H$ intracerebral hemorrhage

untrained user [25], we determined a set of quantifiable indicators for surgical tool-tissue interaction $[10,12,28]$, including hesitations, repetitions/corrections, slowdowns, monitorto-site checks, and added surgical or task-completion time. For comparison during the study, we summed up these indicators in a cumulative hand-eye coordination score (CHECS). Observation and video analysis were performed by the study team. A blinded expert observer was consulted to assess and categorize the microscope recordings as >ocular^ or rexoscope $<$ mode, based on surgical tool handling and microscope command.

\section{Statistical analysis}

All data was analyzed descriptively after completion of the study (SPSS Statistics version 25, 2017, IBM, Armonk/NY, USA). Significant differences between means were determined using a two-sample $t$ test. We further used the MannWhitney $U$ test for analysis of group differences in usability ratings including SUS and SURG-TLX results; Spearman's coefficient has been employed for determining the association of setting alterations (e.g., camera angle) and performance (e.g., hand-eye coordination) during preclinical evaluation. Two-tailed probability values of $P<.05$ were considered statistically significant.

\section{Results}

\section{Preclinical phase}

\section{Setup and ergonomics}

Preferred positions of the external monitor were next to the OR table in direct sight axis to the surgeon with the exoscope positioned overhead. In face-to-face spinal setups the assistant surgeon (S2) has the same camera view as the operating surgeon (S1), which means for $\mathrm{S} 2$ a $180^{\circ}$ rotated image making effective operative assistance impossible. Due to the limited working distance, exoscopic spine procedures are also affected by the surgeon's body height $(<165 \mathrm{~cm}$ optimal) (see Fig. 1a). During exoscopic mode when standing, head posture was
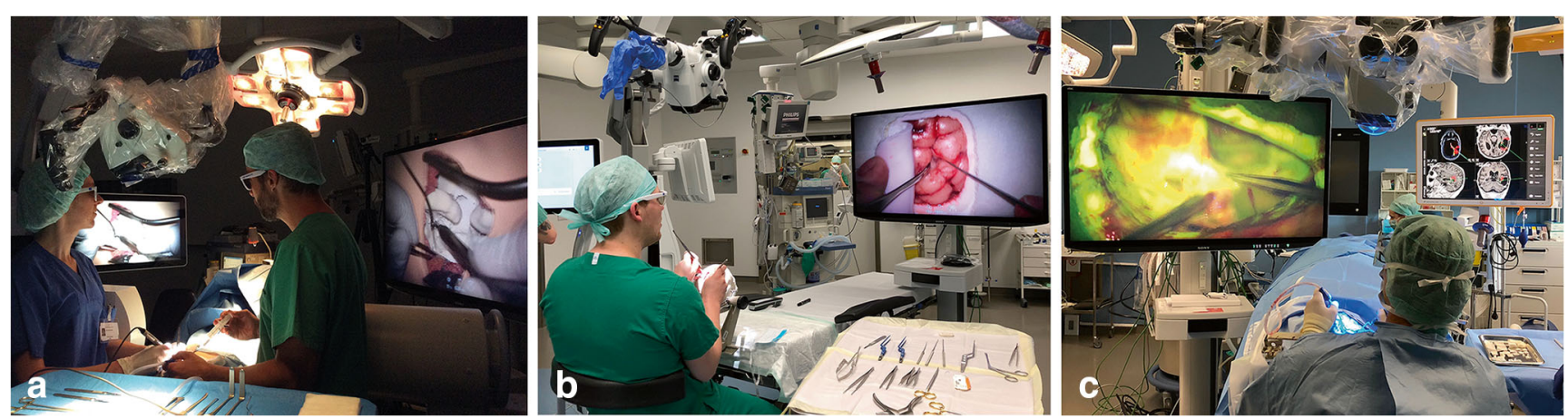

Fig. 1 Preclinical setup: face-to-face scenario in spinal task with two 3D monitors (a); training scenario in cranial task (b); intraoperative setup: >surgical cockpit with 3D4k monitor and navigation screen, camera positioned perpendicular above surgical site (c) 
Table 2 Overview of cases in clinical phase $(n=29)]$

\begin{tabular}{|c|c|c|c|c|c|c|}
\hline Type & Group & Age & Sex & Pathology & Access & Short comment on exoscope utilization \\
\hline Cranial (CE) & - & 49 & $\mathrm{~F}$ & GBM left insular & Ext. pterional & Slowdowns, FCP conflicts, complex case \\
\hline Spinal (CE) & - & 42 & $\mathrm{~F}$ & Lumbar recessus stenosis & Lumbar & Good for in-focus tasks, control via handles faster \\
\hline Cranial (CE) & - & 38 & M & GBM (rec) right temporal & Temporal & Time pressure, camera angulation too lateral \\
\hline Cranial (CE) & - & 54 & $\mathrm{~F}$ & GBM left perisylvian & Temporal & Suboptimal 3D quality, blurring, complex case \\
\hline Cranial (CE) & - & 68 & $\mathrm{~F}$ & Trigeminal nerve decompression & Retrosigmoidal & Improved ergonomics, keyhole-effect in depth \\
\hline Cranial (CE) & - & 24 & M & CSF fistula & Frontal & Improved ergonomics \\
\hline Cranial (CE) & - & 52 & M & aAST (rec) left frontal & Frontal & Camera angulation too lateral \\
\hline Cranial (CE) & - & 58 & M & GBM left parietooccipital/opercular & Parietal & Camera angulation too lateral \\
\hline Cranial (CE) & - & 50 & $\mathrm{~F}$ & MNG right sphenoid wing & Pterional & Comfortable switch with macroscopic vision \\
\hline Cranial (RCT) & OG & 67 & $\mathrm{~F}$ & $\mathrm{CM}$ left precentral & Temporal & - \\
\hline Cranial (RCT) & MG & 70 & $\mathrm{~F}$ & MNG left parietal & Parietooccipital & Conversion for tumor-tissue contrast \\
\hline Cranial (RCT) & MG & 46 & $\mathrm{~F}$ & MNG left precentral & Frontal & Slowdowns, FCP conflicts \\
\hline Cranial (RCT) & MG & 78 & M & GBM right parietal & Parietooccipital & Circumnavigation of resection cavity slow with FCP \\
\hline Cranial (RCT) & OG & 79 & $\mathrm{~F}$ & $\mathrm{aODG}(\mathrm{rec})$ right postcentral & Frontoparietal & - \\
\hline Cranial (RCT) & MG & 38 & $\mathrm{~F}$ & GBM (rec) right frontal & Frontoparietal & $\begin{array}{l}\text { Fluoresceine: tissue contrast suboptimal due } \\
\text { to brightness, but small vessels better detectable }\end{array}$ \\
\hline Cranial (RCT) & OG & 30 & $\mathrm{~F}$ & aAST (rec) left frontal & Frontoparietal & - \\
\hline Cranial (RCT) & MG & 43 & M & aODG (rec) left frontal & Frontoparietal & No multivision info under fluoresceine \\
\hline Cranial (RCT) & OG & 59 & $\mathrm{~F}$ & GBM left suprasylvian & Temporal & - \\
\hline Cranial (RCT) & MG & 32 & M & Met left occipital & Occipital & Handles control instead of FCP \\
\hline Cranial (RCT) & MG & 67 & M & GBM (rec) right temporoparietal & Temporal & Camera angulation too lateral \\
\hline Cranial (RCT) & OG & 57 & M & GBM left frontal & Frontal & - \\
\hline Cranial (RCT) & OG & 56 & M & Met (rec) left parietal parafalcine & Parietal & - \\
\hline Cranial (RCT) & MG & 32 & M & $\mathrm{aODG}$ (rec) left frontal & Frontoparietal & Very good visibility of target structures \\
\hline Cranial (RCT) & OG & 33 & M & aAST left postcentral & Parietal & - \\
\hline Cranial (RCT) & OG & 79 & M & Met right parietooccipital & Occipital & - \\
\hline Cranial (RCT) & MG & 29 & M & MNG right precentral & Frontoparietal & Conversion for tumor-tissue contrast \\
\hline Cranial (RCT) & OG & 71 & $\mathrm{~F}$ & MNG right frontal & Frontoparietal & - \\
\hline Cranial (RCT) & OG & 75 & M & MNG right postcentral & Parietal & - \\
\hline Cranial (RCT) & MG & 52 & M & ODG right frontal & Pterional & Slowdowns; improved ergonomics \\
\hline
\end{tabular}

$a A S T$ anaplastic astrocytoma, $a O D G$ anaplastic oligodendroglioma, $C E$ clinical exploration, $C M$ cavernoma, $C S F$ cerebrospinal fluid, $G B M$ glioblastoma, Met metastasis, $M N G$ meningioma, $M G$ monitor group, $O D G$ oligodendroglioma, $O G$ ocular group, $R C T$ randomized controlled trial, rec recurrence

upright in $95 \%$ and upper body posture was upright in $98.3 \%$ of the time, compared with when sitting, head posture was upright in $87.1 \%$ and upper body posture was upright in $90.8 \%$ of the time (opposed to an average of $70 \%$ upright position in ocular mode for both postures when sitting).

\section{Task performance}

All participants were able to complete the tasks. Ten of 12 surgeons $(83.3 \%)$ had a higher rate of movement hesitations and corrections in monitor-based performance. Monitor-tosite angles $>45^{\circ}$ caused high discomfort. In monitor-to-site angles $<45^{\circ}$, we observed a linear increase of hesitations, corrections, and monitor-to-site checks (angle $0-10^{\circ}$ : mean
CHECS 14.6; angle >10: mean CHECS 20.7). Furthermore, we saw differences between more paramedian (frontoparietal) and more lateral (pterional) approaches (see Fig. 2) including a positive correlation between more lateral camera inclination and impact on hand-eye coordination $\left(r_{\mathrm{s}}=\right.$ $0.756, P=.01)$.

\section{Usability}

Overall participant ratings across all 30 questionnaire items were good (median 2.0); only item $12>$ system monitor usability in $\mathrm{S} 2$ < was ranked distinctly lower with median 4.0 (not acceptable) (see supplementary material, Table 3 ). Free text comments highlighted improved team communication and 
Fig. 2 Synopsis of effects on hand-eye coordination in cranial task performance compared for frontoparietal (light gray) and pterional (dark gray) approaches (participants $n=9$ ); plotted on the horizontal axis are numbers of hesitations, corrections, and direct sight control plus overtime needed for task completion in minutes

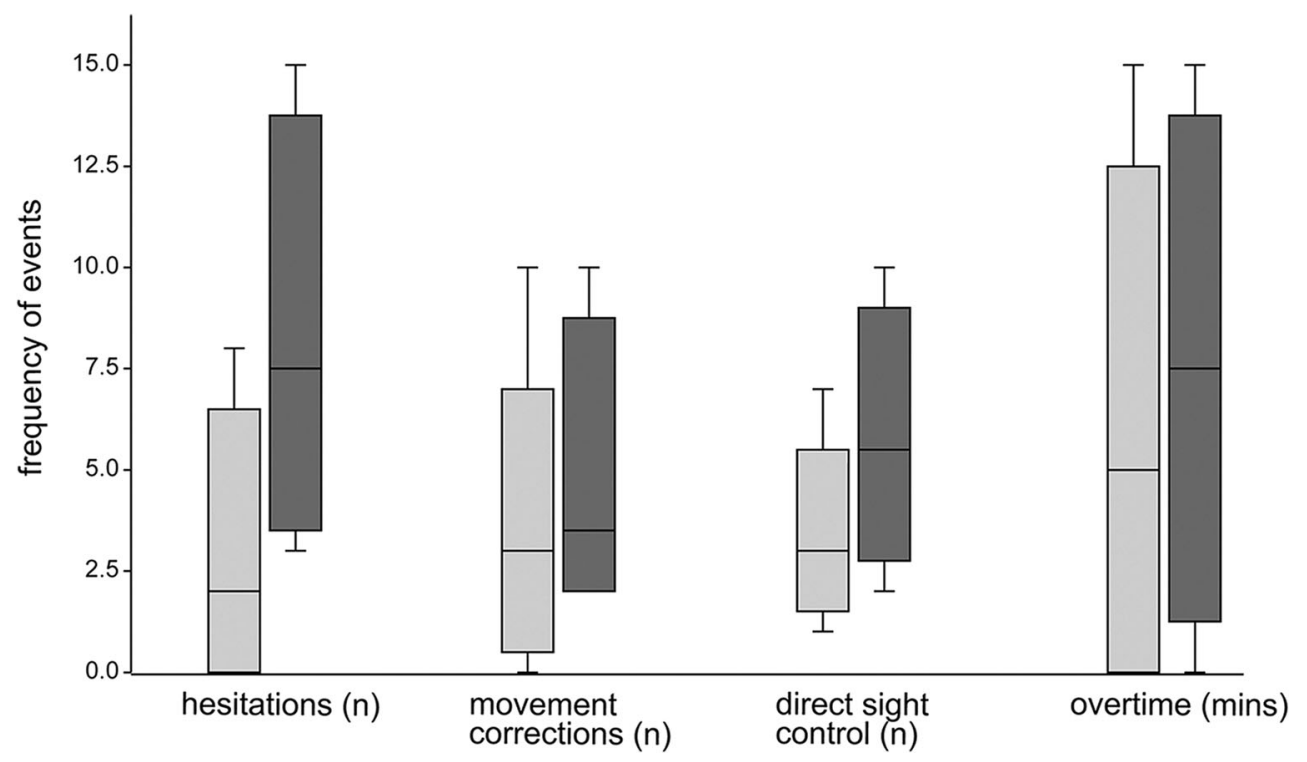

ergonomics on the one hand, and impaired hand-eye coordination and complicated hand-free camera control on the other hand (see supplementary material, Table 4).

\section{Image quality}

Image quality assessment focused on visual interferences (transmission delay $0 \%$, monitor reflection $26.7 \%$, image blurring 20\%), satisfactory depth vision (80\%), contrast $(86.7 \%)$, and screen resolution (100\%) (see Fig. 3). In 5 participants $(41.7 \%)$, image quality has been rated inferior to ocular mode due to quality discrepancies between in-focus and off-focus areas.

\section{Clinical phase}

All participants tested with the Frisby Pocket Test had a stereoacuity of $\leq 75 \mathrm{~s}$ arc, thus showing no indication of altered stereovision. Due to current limitations in spinal interventions, the focus of clinical case evaluation (CE) was on various types of cranial surgeries (see Table 2 ).

\section{Clinical data}

Tumor pathologies included 25\% glioblastoma, 25\% meningioma, $15 \%$ anaplastic oligodendroglioma, $15 \%$ metastasis, $10 \%$ anaplastic astrocytoma, 5\% oligodendroglioma, and $5 \%$ cavernoma (mean patient age $55 \pm 18$ years, range 29 79; $60 \%$ male; $65 \%$ initial diagnosis; $55 \%$ left-sided lesions; $50 \%$ frontal, $30 \%$ parietal, $10 \%$ temporal, $10 \%$ occipital lesions; for legend of abbreviations and further information, see Table 2). Planned complete (CR) or gross total resection (GTR) could be achieved in $94 \%$ of cases, based on imaging results. Ten percent of cases $(n=2)$ had minor intraoperative complications (increased blood loss, subacute PICA infarction), which were however limited to the ocular group. Postoperative functional outcome was $80 \%$ idem, $10 \%$ impairment, $5 \%$ transient deficit, and $5 \%$ improvement.
Fig. 3 Dimensions of image quality satisfaction (\%) present in digital visualization, compared between preclinical (light gray) and clinical (dark gray) settings

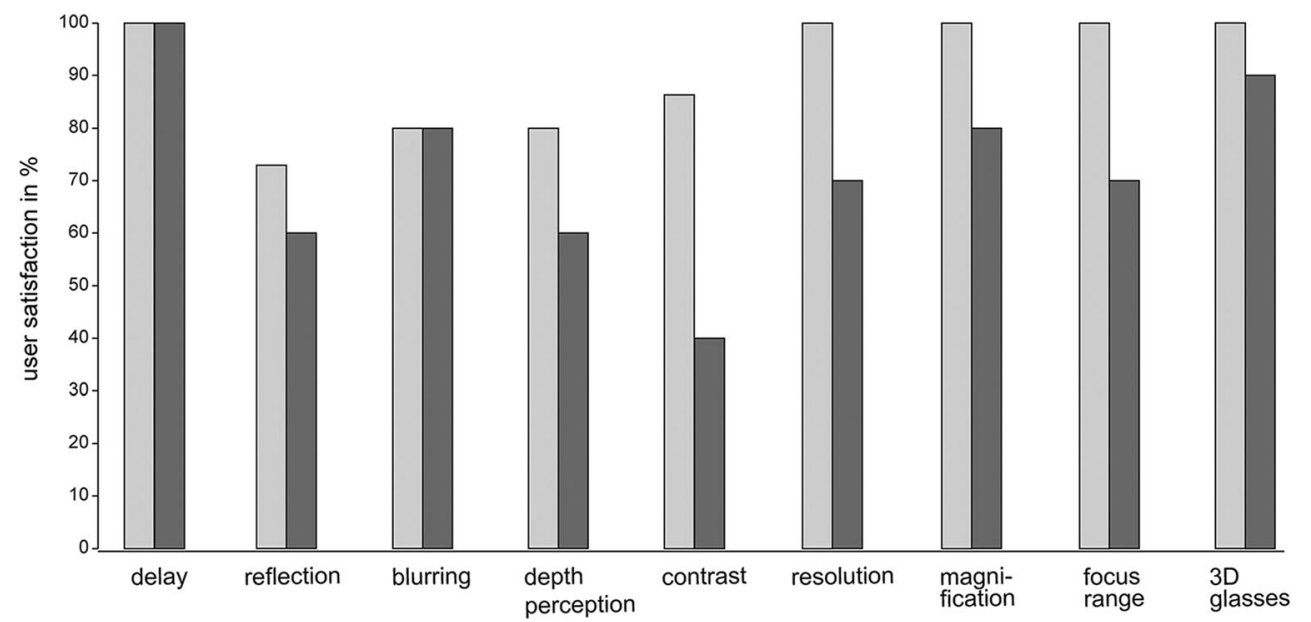


Patient characteristics per group included a mean age of 61 years, $50 \%$ male, $40 \%$ eloquent location, $30 \%$ recurrence, $10 \%$ new permanent deficits, and $60 \%$ malignant/WHO grade III or higher tumors (OG) vs. a mean age of 49 years, $70 \%$ male, $10 \%$ eloquent location, $40 \%$ recurrence, $10 \%$ new permanent deficits, and $60 \%$ malignant/WHO grade III or higher tumors (MG). Fluorescence guidance has been used according to suspected pathology in 40\% (OG) and 50\% (MG) of cases respectively.

\section{Setup and ergonomics}

Setup integration has been achieved successfully in different neurosurgical ORs (see Fig. 1c). Mean monitor distance and angle were $180 \pm 20 \mathrm{~cm}$ and $10 \pm 10^{\circ}$. A right-sided position of the external monitor was preferable whenever a surgical assistant (S2) was present (see Fig. 4). The alignment of working and viewing direction (i.e., monitor-to-site angles $<10^{\circ}$ ) has been identified as a precondition (see Fig. 4). Overall surgical ergonomics when sitting improved in $\mathrm{MG}$ compared with OG (mean percentage of upright head posture during resection $\mathrm{MG} 72 \%$ vs. OG 52\%, 95\% CI: $-52.2-12.2$; $P=.21$; mean percentage of upright upper body posture during resection $\mathrm{MG} 80 \%$ vs. OG $56 \%, 95 \% \mathrm{CI}$ : -44.6 to -3.4 ; $P=.03)$.
FCP integration turned out challenging in surgeries with two or more foot control panels in active use. Clinical user ratings showed a median score of 3.0 (acceptable) for all items with the following items rated difficult: >off-focus tasks $<, ~>S 2$ integration « and >autofocus` (median 4.0). Highest-ranked items were >ergonomics` and >in-focus tasks` (median 2.0).

\section{Workflow}

There was no significant added surgical time in MG (mean overall surgical time: MG $220 \pm 61$ mins, 95\% CI: 177-264, vs. OG $236 \pm 67 \mathrm{mins}, 95 \% \mathrm{CI}: 188-284 ; P=.63$; mean resection time $\mathrm{MG} 45 \pm 23$ mins, 95\% CI: 28-61, vs. OG $34 \pm$ 16 mins, $95 \%$ CI: $22-45 ; P=.28)$. The conversion rate in $\mathrm{MG}$ was $50 \%$ with case-dependent issues in lateral camera inclination and visual tissue differentiation. There was no conversion from OG to MG. Average monitor time in MG was $30 \pm$ 20 mins (68\% of MG mean resection time). Added preparation time was 10 mins on average.

\section{Usability}

An impact of exoscopic surgery on surgical hand-eye coordination has been observed in the majority of cases $(70 \%$ hesitations, $80 \%$ corrections/repetitions, and $80 \%$ slowdowns) during the first $7.5 \pm 2.5$ mins of resection. The blinded expert

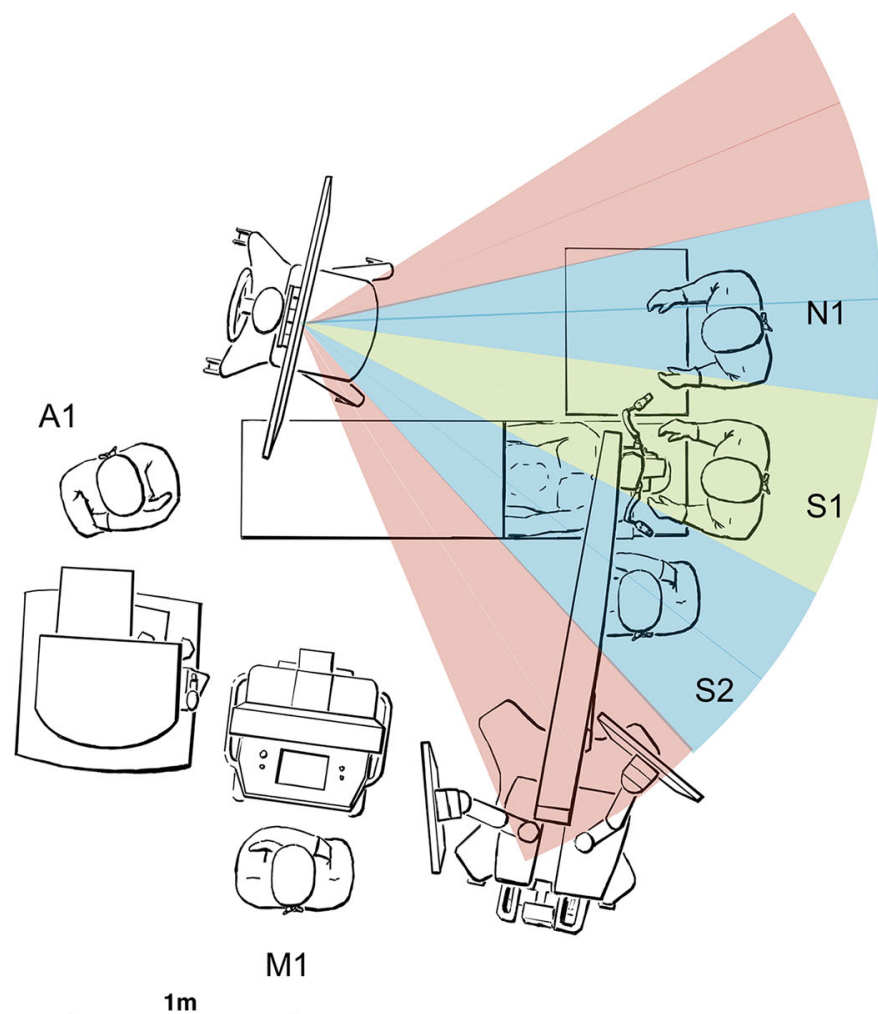

\begin{tabular}{|c|l|}
\hline angle & effect on working position \\
\hline $0^{\circ}$ & optimal \\
\hline$>10^{\circ}$ & noticeable \\
\hline$>30^{\circ}$ & uncomfortable \\
\hline$>45^{\circ}$ & impossible \\
\hline
\end{tabular}

Fig. 4 Intraoperative setup in cranial interventions with surgical assistant (S2), scrub nurse (N1), neuromonitorist (M1), and anesthesiologist (A1) showing effects of monitor-to-site angle on surgical performance 
rater was able to identify $68 \%$ of the case modes correctly, only being mistaken in the cases of the one expert user who did the most exoscopic surgeries in this study. FCP usability has been rated lower in the clinical phase (median preclinical $2.0=$ good vs. clinical $3.5=$ difficult). It was considered the most useful for small position refinements. SUS (score range $0-100$, high score indicates high usability) showed significant group differences in items $1>$ readiness to use < (median MG 3.5 vs. OG 5.0; $P<.001$ ), 3 >usability< (median MG 4.0 vs. OG 5.0; $P<.001$ ), 9 >confidence (median MG 4.0 vs. OG $5.0 ; P=.02$ ) and overall score (mean MG $76.3 \pm 22,5$ vs. OG $93.5 \pm 9,1$, 95\% CI: 0.53-33.97; $P=.04$ ) (see Fig. 5a). SURG-TLX (score range $6-120$, high score indicates high surgical workload) showed significant group differences with increase of overall score (mean MG $47.2 \pm 19.2$ vs. OG $31.8 \pm 12.3,95 \%$ CI: 30.51 to $-0.29 ; P=.04$ ) and item 6 >distraction $<$ (median MG 4.5 vs. OG $3.0 ; P=.04$ ) in MG (see Fig. 5 b).

\section{Image quality}

MG satisfaction ratings in image quality were lower compared with ocular mode (screen resolution $70 \%$, image contrast $40 \%$, depth perception $60 \%$, magnification $80 \%$, focus range 70\%, 3D glasses 90\%; see Fig. 3). Main shortcomings stated were color accuracy, illumination, and image contrast when applied to tumor-tissue differentiation in detection of tumor borders.

\section{Discussion}

The principle novel findings of our study comprise the overall clinical feasibility, safety, and ergonomic advantageousness of

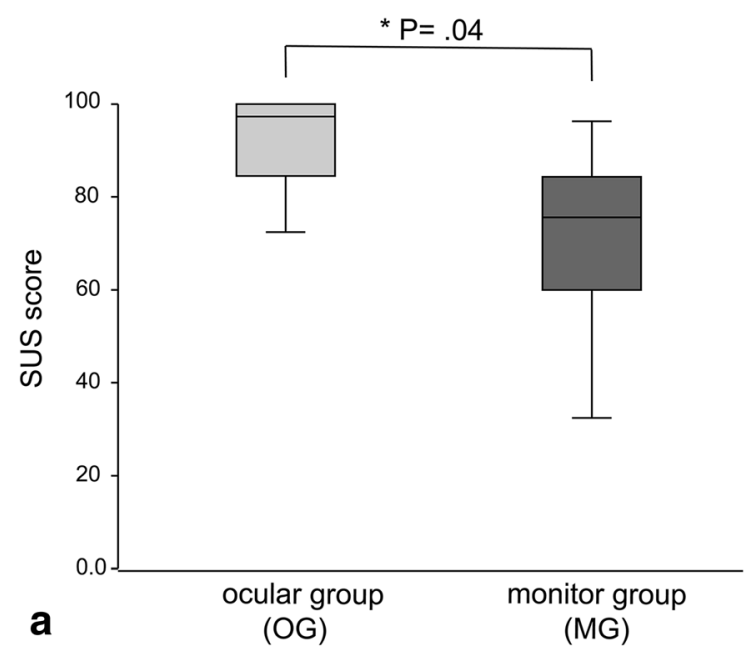

Fig. 5 System Usability Scale (SUS) (a) and Surgery Task Load Index (SURG-TLX) (b) across all cases compared for both groups (OG, MG), showing an overall decreased mean usability and an increased mean
3D4k hybrid exoscopic surgery in supratentorial brain tumors. We further analyzed shown restrictions in usability and visualization quality that limit the utilization of current generation exoscopes in more complex neurosurgical interventions.

\section{Clinical usability and eligible cases}

In cranial interventions, our findings support a monitor distance of $180 \mathrm{~cm}$ and angle of $0-10^{\circ}$ for the main surgeon with the monitor positioned on the scrub nurse side of the OR table.

By combining assessments of usability and task load, we could identify areas where further action (training, improved control devices) is needed in order to achieve user satisfaction (technological confidence) and reduce added contextual burden (distraction) for the surgeon. The critically limiting factor in device usability was the FCP controlling exoscope robotics. The full potential of exoscopic surgery will only be exploited once intuitive hand-free controls of the camera position are implemented. All participants agreed that with the greater field of view in exoscope mode, effects of distraction are more relevant than in ocular mode. This correlates with the significantly increased SURG-TLX score in item 6 >distraction<. In more remote positions of the external monitor, the effect of distraction increases.

Due to an interdependence of increasingly lateral camera angles and restricted visibility of structures in the depth of the resection cavity, frontoparietal and pterional approaches were better suited for exoscopic surgery than retrosigmoidal or suboccipital approaches. Supratentorial brain tumor cases that do not require a small surgical corridor are most suited for exoscopic surgery with a hybrid visualization system. Our use cases equal a level II complexity [11] with the option to increase surgical complexity with a long-term learning curve.

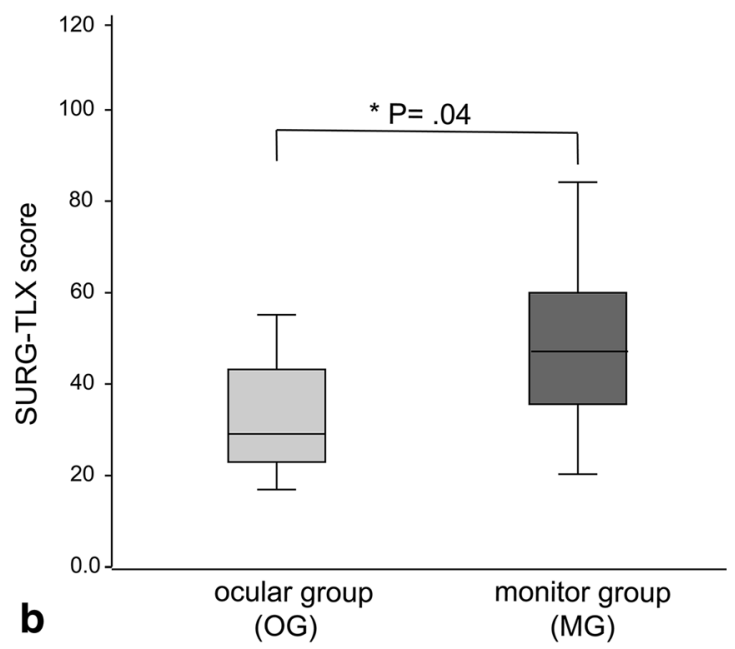

workload in MG respectively with single users achieving comparable scores in both operating modes 
Due to current limitations (S2 integration, S1 body height), spinal interventions are not suitable for exoscopic surgery with this system.

\section{Impact on performance}

Most exoscope studies report improved ergonomics for the operating surgeon (see Table 1). However, the surgeon's body posture is determined by the patient's position, surgical access, and viewing angle to the surgical site. Still, we could show that, in direct comparison with the ocular mode, the exoscope is in many cases ergonomically favorable.

Hand-eye coordination is clearly affected in exoscope mode. During familiarization with the technology, similar issues could be observed as in 2D exoscopes and endoscopes: many surgeons used initially both hands in order to understand depth and range of their instrument handling, guiding one instrument with another. The adjustment time decreases with experience. Earlier reports [20] stated an adaptation period of approx. 60 minutes for hand-eye coordination in monitor-based 2D visualization-less, if the surgeon was trained in neuroendoscopy. Acclimatization time needed for optimal dexterity was further related to the depth of field.

\section{Quality of visualization}

Image quality was rated more critically during the clinical study phase (see Fig. 3) when the requirements of image quality became case-specific. In exoscope mode, small focus ranges in the depth of a resection cavity limit in-focus working noticeably compared with the optic mode where physiologic eye accommodation can actually compensate for off-focus actions. Thus, the actual (in-focus) working area in deepseated structures turns out smaller. The field of view is cut to fit the rectangular monitor, requiring more frequent camera position adjustments during surgery. Regarding tissue differentiation in brain tumor interventions, there are different positions in the literature [23] stating better, equal, or worse quality compared with optical microsurgery. However, image contrast including color accuracy was rated the lowest in this study; beside detection of tumor-tissue borders, fast visual identification of small bleeding vessels can be challenging. The utilization of a hybrid visualization system like the one investigated in this study can help adjust workflows and gain experience in more complex microneurosurgical tasks before switching to a standalone exoscope. Until completion of this paper, one other study on the same system has been published [4] presenting results of a comparative preclinical assessment of microvascular anastomosis. The authors conclude that the 3D4k exoscope allows for neurovascular task completion; however, in their evaluation, size and width of the field of view as well as sharpness and resolution at high magnification were still superior in ocular mode. We claim that established minimum visual requirements such as illumination, magnification, depth vision, and detail/overview are inadequate for high-quality exoscopic microneurosurgery. Rather, all image settings including brightness, color filters, contrast, resolution, and sharpness should be assessed and further investigated in detail, as they define the visual expectation in the trained expert neurosurgeon.

\section{Limitations of this study}

This study has been designed to investigate the hybrid exoscope under clinical routine conditions in the neurosurgical OR. As a consequence of clinical feasibility and intraoperative workflow, we did not employ any advanced quantitative measuring systems for assessment of technical performance, such as eye and motion tracking devices. Higher numbers of cases and participating surgeons are required for further investigation of eligible procedures in exoscopic microneurosurgery. We would also like to note that microscope visualization and expert command of the technology will be even more critical in complex surgical cases. During the course of this study, an updated system with optimized image quality parameters was released (KINEVO 900 version $1.5)$ that could not be taken into consideration.

\section{Conclusions}

3D4k exoscopic surgery is feasible in clinical routine with clearly defined limitations. Depending on surgical access and case, neurosurgical routine tasks can be performed unrestrictedly. Major advantages of the exoscope are improved ergonomics, an unblocked primary surgical field of vision and a shared 3D view for the complete surgical team offering increased educational value. Major limitations concern interventions through small surgical corridors and the high frequency of camera adjustments in monitor mode. Further disadvantages include a temporally impaired hand-eye coordination, imperfect hand-free control, increased spatial requirements in the OR, and limitations in image quality (brightness, contrast, color accuracy). In our case series, we observed a conversion rate of $50 \%$ from exoscope to microscope. Based on our results, we cannot recommend abandoning the established standard at this point. In future exoscopic neurosurgery, improvements in monitor, camera, and image filter technology are indispensable for routine application.

Author's contributions All authors contributed to the study conception and design. Material preparation, data collection, and analysis were performed by Anna Roethe and Thomas Picht. The first draft of the manuscript was written by Anna Roethe and all authors commented on previous versions of the manuscript. All authors read and approved the final manuscript. 
Funding information Open Access funding provided by Projekt DEAL. This work has in part been funded by the Cluster of Excellence Image Knowledge Gestaltung - an Interdisciplinary Laboratory (German Research Foundation, research grant number EXC 1027/1). Carl ZEISS Meditec AG (Oberkochen, Germany) provided financial support in the form of workshop supplies and technological support.

\section{Compliance with ethical standards}

Ethics statement All procedures performed in studies involving human participants were in accordance with the ethical standards of the institutional research committee (Charité Ethics Committee, reference number EA2/081/18) and with the 1964 Helsinki declaration and its later amendments or comparable ethical standards. Informed consent was obtained from all individual participants included in the study.

Conflict of interest All authors certify that they have no affiliations with or involvement in any organization or entity with any financial interest (such as honoraria; educational grants; participation in speakers' bureaus; membership, employment, consultancies, stock ownership, or other equity interest; and expert testimony or patent-licensing arrangements), or non-financial interest (such as personal or professional relationships, affiliations, knowledge or beliefs) in the subject matter or materials discussed in this manuscript.

Open Access This article is licensed under a Creative Commons Attribution 4.0 International License, which permits use, sharing, adaptation, distribution and reproduction in any medium or format, as long as you give appropriate credit to the original author(s) and the source, provide a link to the Creative Commons licence, and indicate if changes were made. The images or other third party material in this article are included in the article's Creative Commons licence, unless indicated otherwise in a credit line to the material. If material is not included in the article's Creative Commons licence and your intended use is not permitted by statutory regulation or exceeds the permitted use, you will need to obtain permission directly from the copyright holder. To view a copy of this licence, visit http://creativecommons.org/licenses/by/4.0/.

\section{References}

1. Bakhsheshian J, Strickland BA, Jackson C, Chaichana KL, Young R, Pradilla G, Chen JW, Bailes J, Zada G (2018) Multicenter investigation of channel-based subcortical trans-sulcal exoscopic resection of metastatic brain tumors: a retrospective case series. Oper Neurosurg (Hagerstown). https://doi.org/10.1093/ons/opy079

2. Beez T, Munoz-Bendix C, Beseoglu K, Steiger H-J, Ahmadi SA (2018) First clinical applications of a high-definition three-dimensional exoscope in pediatric neurosurgery. Cureus 10(1):e2108

3. Belloch JP, Rovira V, Llácer JL, Riesgo PA, Cremades A (2014) Fluorescence-guided surgery in high grade gliomas using an exoscope system. Acta Neurochir 156(4):653-660

4. Belykh E, George L, Zhao X, Carotenuto A, Moreira LB, Yağmurlu K, Bozkurt B, Byvaltsev VA, Nakaji P, Preul MC (2018) Microvascular anastomosis under 3D exoscope or endoscope magnification: a proof-of-concept study. Surg Neurol Int 9:115

5. Biddle M, Hamid S, Ali N (2014) An evaluation of stereoacuity (3D vision) in practising surgeons across a range of surgical specialities. Surgeon 12(1):7-10

6. Birch K, Drazin D, Black KL, Williams J, Berci G, Mamelak AN (2014) Clinical experience with a high definition exoscope system for surgery of pineal region lesions. J Clin Neurosci 21(7):12451249
7. Boutron I, Altman DG, Moher D, Schulz KF, Ravaud P, for the CONSORT NPT Group (2017) CONSORT statement for randomized trials of nonpharmacologic treatments: a 2017 update and a CONSORT extension for nonpharmacologic trial abstracts. Ann Intern Med 167(1):40

8. Brooke J (1996) SUS: a quick and dirty usability scale. In: Jordan PW, Thomas B, Weerdmeester BA, McClelland IL (eds) Usability evaluation in industry. Taylor \& Francis, London, pp 189-194

9. Gassie K, Wijesekera O, Chaichana KL (2018) Minimally invasive tubular retractor-assisted biopsy and resection of subcortical intraaxial gliomas and other neoplasms. J Neurosurg Sci. https://doi.org/ 10.23736/S0390-5616.18.04466-1

10. Ghasemloonia A, Maddahi Y, Zareinia K, Lama S, Dort JC, Sutherland GR (2017) Surgical skill assessment using motion quality and smoothness. J Surg Educ 74(2):295-305

11. Gonen L, Chakravarthi SS, Monroy-Sosa A, Celix JM, Kojis N, Singh M, Jennings J, Fukui MB, Rovin RA, Kassam AB (2017) Initial experience with a robotically operated video optical telescopic-microscope in cranial neurosurgery: feasibility, safety, and clinical applications. Neurosurg Focus 42(5):E9

12. Harada K, Morita A, Minakawa Y, Baek YM, Sora S, Sugita N, Kimura T, Tanikawa R, Ishikawa T, Mitsuishi M (2015) Assessing microneurosurgical skill with medico-engineering technology. World Neurosurg 84(4):964-971

13. Jackson C, Gallia GL, Chaichana KL (2017) Minimally invasive biopsies of deep-seated brain lesions using tubular retractors under exoscopic visualization. J Neurol Surg A Cent Eur Neurosurg 78(6):588-594

14. Kassam AB, Chakravarthi SS, Celix J, Fukui M, Jennings J, Walia S, Rovin RA (2016) 113 initial experience with an image-guided robotically positioned optical platform for aneurysm surgery. Neurosurgery 63(CN_suppl_1):147-148

15. Klinger DR, Reinard KA, Ajayi OO, Delashaw JB (2018) Microsurgical clipping of an anterior communicating artery aneurysm using a novel robotic visualization tool in lieu of the binocular operating microscope: operative video. Oper Neurosurg (Hagerstown) 14(1):26-28

16. Krishnan KG, Schöller K, Uhl E (2017) Application of a compact high-definition exoscope for illumination and magnification in high-precision surgical procedures. World Neurosurg 97:652-660

17. Kwan K, Schneider JR, Du V, Falting L, Boockvar JA, Oren J, Levine M, Langer DJ (2018) Lessons learned using a highdefinition 3-dimensional exoscope for spinal surgery. Oper Neurosurg (Hagerstown). https://doi.org/10.1093/ons/opy196

18. Mamelak AN, Danielpour M, Black KL, Hagike M, Berci G (2008) A high-definition exoscope system for neurosurgery and other microsurgical disciplines: preliminary report. Surg Innov 15(1):38-46

19. Mamelak AN, Nobuto T, Berci G (2010) Initial clinical experience with a high-definition exoscope system for microneurosurgery. Neurosurgery 67(2):476-483

20. Mamelak AN, Drazin D, Shirzadi A, Black KL, Berci G (2012) Infratentorial supracerebellar resection of a pineal tumor using a high definition video exoscope (VITOM®). J Clin Neurosci 19(2):306-309

21. Moisi MD, Hoang K, Tubbs RS et al (2017) Advancement of surgical visualization methods: comparison study between traditional microscopic surgery and a novel robotic optoelectronic visualization tool for spinal surgery. World Neurosurg 98:273-277

22. Nishiyama K (2017) From exoscope into the next generation. J Korean Neurosurg Soc 60(3):289-293

23. Oertel JM, Burkhardt BW (2017) Vitom-3D for exoscopic neurosurgery: initial experience in cranial and spinal procedures. World Neurosurg 105:153-162

24. Piquer J, Llácer JL, Rovira V, Riesgo P, Rodriguez R, Cremades A (2014) Fluorescence-guided surgery and biopsy in gliomas with an exoscope system. Biomed Res Int 2014:207974 
25. Rossini Z, Cardia A, Milani D, Lasio GB, Fornari M, D'Angelo V (2017) VITOM 3D: preliminary experience in cranial surgery. World Neurosurg 107:663-668

26. Sack J, Steinberg JA, Rennert RC, Hatefi D, Pannell JS, Levy M, Khalessi AA (2018) Initial experience using a high-definition 3dimensional exoscope system for microneurosurgery. Oper Neurosurg (Hagerstown) 14(4):395-401

27. Shirzadi A, Mukherjee D, Drazin DG, Paff M, Perri B, Mamelak AN, Siddique K (2012) Use of the video telescope operating monitor (VITOM) as an alternative to the operating microscope in spine surgery. Spine 37(24):E1517-E1523

28. Sugiyama T, Lama S, Gan LS (2018) Forces of tool-tissue interaction to assess surgical skill level. JAMA Surg 153(3):234-242
29. Takahashi S, Toda M, Nishimoto M, Ishihara E, Miwa T, Akiyama T, Horiguchi T, Sasaki H, Yoshida K (2018) Pros and cons of using ORBEYETM for microneurosurgery. Clin Neurol Neurosurg 174: 57-62

30. Wilson MR, Poolton JM, Malhotra N, Ngo K, Bright E, Masters RSW (2011) Development and validation of a surgical workload measure: the surgery task load index (SURG-TLX). World J Surg 35(9):1961-1969

Publisher's note Springer Nature remains neutral with regard to jurisdictional claims in published maps and institutional affiliations.

\section{Affiliations}

\section{Anna L. Roethe ${ }^{1,2}$ (D) Philipp Landgraf ${ }^{3} \cdot$ Torsten Schröder $^{3} \cdot$ Martin Misch $^{1} \cdot$ Peter Vajkoczy $^{1} \cdot$ Thomas Picht $^{1,2}$ \\ 1 Department of Neurosurgery, Charité-Universitätsmedizin Berlin, \\ 3 Department of Anesthesiology and Operative Intensive Care Charitéplatz 1, 10117 Berlin, Germany \\ Medicine, Charité-Universitätsmedizin Berlin, Berlin, Germany}

2 Interdisciplinary Laboratory Image Knowledge Gestaltung, Humboldt-Universität zu Berlin, Berlin, Germany 